\title{
An efficient traffic contention and control mechanism to improve QoS in heterogeneous wireless sensor networks
}

\author{
G. Kavitha, P.Latchoumy, A.Sonya
}

Department of Information Technology, BSA Crescent Institute of Science and Technology, India

\begin{tabular}{l} 
Article Info \\
\hline Article history: \\
Received Dec 20, 2019 \\
Revised Mar 22, 2020 \\
Accepted Apr 5, 2020 \\
\hline
\end{tabular}

\section{Keywords:}

Congestion control

Efficient node

Network topology

QoS

Transmission delay

\begin{abstract}
Heterogeneous Wireless Sensor Networks (HWSN) gathers information from a cooperative network. In HWSN, the sensor nodes are scattered and the major challenges are topology control, battery optimization, packet loss and link lifetime. The existing techniques do not concentrate on all the mentioned issues. The objective of this work is to provide congestion-free data transfer with higher throughput and increased packet delivery ratio. In the proposed methodology, three protocols are designed and developed, namely, Hop by Hop Rate Adjustment Protocol (HHRA), Energy Efficient Data Transfer Protocol (EEDT) and Alternative Routing Congestion Control Protocol (ARCC). The HHRA protocol senses the traffic in the channel and adjusts the transmission rate accordingly to avoid congestion. Secondly, the EEDT protocol is used to find specific nodes that are more efficient and transfer packets through those nodes to improve throughput. The ARCC protocol is used to redirect the path of transmission during the occurrence of congestion. Thus, the proposed traffic contention and control mechanisms ensures congestion free transmission and increases the packet delivery ratio by $23 \%$ and average throughput by $20 \%$ compared to the Dynamic Contention Window based Congestion Control (DCWCC) algorithm.
\end{abstract}

Copyright $(2020$ Institute of Advanced Engineering and Science. All rights reserved.

Corresponding Author:

G. Kavitha,

Department of Information technology,

BSA Crescent Institute of Science and Technology, India.

Email: gkavitha.78@gmail.com

\section{INTRODUCTION}

Heterogeneous Wireless Sensor Networks (HWSNs) is considered as one of the growing technologies. The sensor nodes are usually scattered, and it is not necessary to pre-determine the position of the sensor nodes in the network. HWSNs are the self-configuring network of tiny nodes connected by wireless links and communicate with a sink node (base station) [1]. Moreover, WSNs have limited bandwidth and limited battery power.

Sensor nodes collect measurements of interest over a given space and make them available to external systems and networks at sink nodes $[2,3]$. The power saving techniques are commonly implemented to increase the independence of the individual nodes and this technique makes the nodes to sleep most of the time. This can be balanced with low power communications, which usually lead to multi-hop data transmission from sensor nodes to sink nodes and vice versa. The drawbacks of HWSNs are energy conservation, congestion and contention control, reliable data dissemination, security and management.

The topology in wireless networks varies from time to time and topology control has a great impact on performance. The existing topology control methods stimulate the cooperation between nodes and concentrate on only one issue at a time either energy consumption or link lifetime and further is not adaptive to the variation of the topology. Adaptation to topology chages is difficult due to insertion of new nodes, broken links, battery power and scheduling [4]. 
In WSN, the energy consumption by the nodes can be minimized by choosing hierarchical routing protocols [5]. As the energy on the node is limited, energy efficient routing methods are provided to route the packets to the destination [6]. The energy of the sensor nodes can be decreased by long transmissions in a multi-hop network [7]. The energy saving methods in WSN has to consider the QoS parameters like delay, throughput and network life time [8,9].

The performance of the WSN decreases due to packet collisions, network congestion and packet loss. Adaptive congestion control mechanisms based on the node rank is used to find alternate path for transmission of data packets [10]. Congestion has a significant impact on QoS parameters like packet delivery ratio (PDR), end-toend delay and energy consumption in wireless nodes. There is a performance degradation of the QoS in WSN due to the increase in the probability of failure of sensor node in the network [11].

Hence, in this work, an efficient traffic contention and control mechanism is designed using three protocols namely, Hop by Hop Rate Adjustment protocol (HHRA), Energy Efficient Data Transfer Protocol (EEDT) and Alternative Routing for Congestion Control Protocol (ARCC) to overcome the above-stated problems. The proposed algorithms mitigate the traffic congestion in a heterogeneous network, increases the efficiency of a sensor node by optimizing its battery power and decreases the hop counts by transmitting data through selective nodes and thereby minimize the transmission delay.

The rest of the paper is organized as follows. Section 2 presents the related literature and Section 3 presents the traffic contention and control mechanism for HWSN. The results of simulation are presented in Section 4. Finally, Section 5 gives the conclusions and provides directions for future work.

\section{LITERATURE REVIEW}

An adaptive multi-objective optimization framework is presented for coverage and topology control in heterogeneous wireless sensor networks [12]. The multi-objective optimization approach of the proposed framework can simultaneously optimize several conflicting issues such as the number of active sensor nodes, the coverage rate of the monitoring area and balanced energy consumption while maintaining the network connectivity.

Gui and Zeng proposed the network lifetime augmentation in remote multi-bounce systems [13]. The Lifetime and Delay based restricted Topology Control calculation is used to build the topology for every hub. The authors have presented a Distributed Logical Neighbor Adjustment calculation, by which every hub modifies its coherent neighbors and these nodes drain their energy reasonably. The drawback of this topology control is that it drags out the lifetime of the system.

Quiming Liu et. al has considered the influence of network topology on the delay analysis in a largescale wireless Ad-hoc network [14]. The packet transmission delay is an important parameter to evaluate the network QoS performance. Due to the heterogeneity of network topology, the percolation theorem is used to divide the topology into a grid and a new routing policy is implemented to reduce end to end stochastic delay.

Chen et.al proposed a deployment and topology control method for heterogeneous sensor nodes with different communication and sensing range [15]. The behavior of sensor nodes is approximated using irregular sensor model. A cost model is also used to evaluate the deployment cost of heterogeneous WSN. The proposed method achieves higher coverage rates but has higher deployment costs for deploying sensor nodes.

The QoS aware routing protocol should ensure reliability and timely delivery of critical data [16]. A multi criteria decision making model to analyze the performance of different QoS paprameters is discussed in [17]. Gui and Zhou have proposed topology control plans to accomplish both transmission control and network capacity [18]. But these methods have degraded the network performance particularly when k-hop neighborhood size and network density is incremented. For any network application, it is necessary that the network capacity or energy consumption has to be given more consideration to attain appropriate QoS.

The major congestion control methods are categorized into traffic control protocols and resource control protocols [19]. Congestion are handled by conventional methods like network resource management and traffic control [20] and also by soft computing methodologies like feed forward neural networks [21]. A distributed congestion control mechanism using adaptive traffic mechanism to provide fair packet delivery is discussed by Daniela et.al [22]. The equality in packet delivery is done by adjusting the congestion control parameter like buffer size based on the node position. The drawback is that if packets arrive after the given expiration time, there is an increase in the packet loss even if the buffer size is increased.

\section{TRAFFIC CONTENTION AND CONTROL}

The network model for efficient traffic contention and control mechanism represents the order of execution of the three protocols namely, Hop by Hop Rate Adjustment protocol (HHRA), Energy Efficient Data Transfer Protocol (EEDT) and Alternative Routing for Congestion Control Protocol (ARCC). The network model consists of $\mathrm{N}$ sensor nodes randomly distributed within a coverage area and a sink node [21]. 
A sample network containing 20 nodes with their range is shown in the Figure 1 . It is shown that the range of efficient nodes is higher than that of the normal nodes. The data packets can be sent to the destination node in just 4 hops through the e-nodes.

The proposed architectural diagram for Traffic Contention and Control mechanism is shown in Figure 2. The input data from multiple sensor nodes is fed to the traffic regulator and the data traffic is regulated by calculating the Node ranks of the sensor nodes at each stage. An average throughput value is set. When the node rank value crosses the threshold ' $t$ ', the nodes set a congestion bit in every packet it forwards. On receiving the packets, the neighbouring node calculates the Rate Adjustment Feedback (RAF) and sends it upstream towards the source. Using the RAF value, the transmission rate is adjusted to prevent congestion.

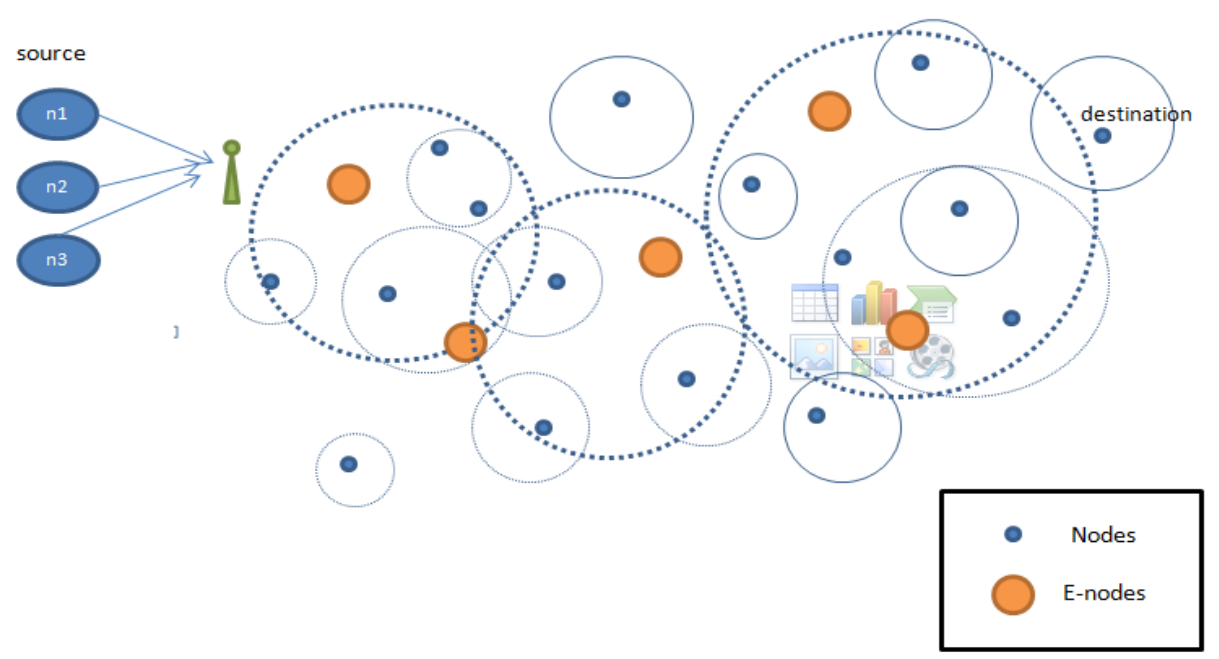

Figure 1. Sensor nodes in a network

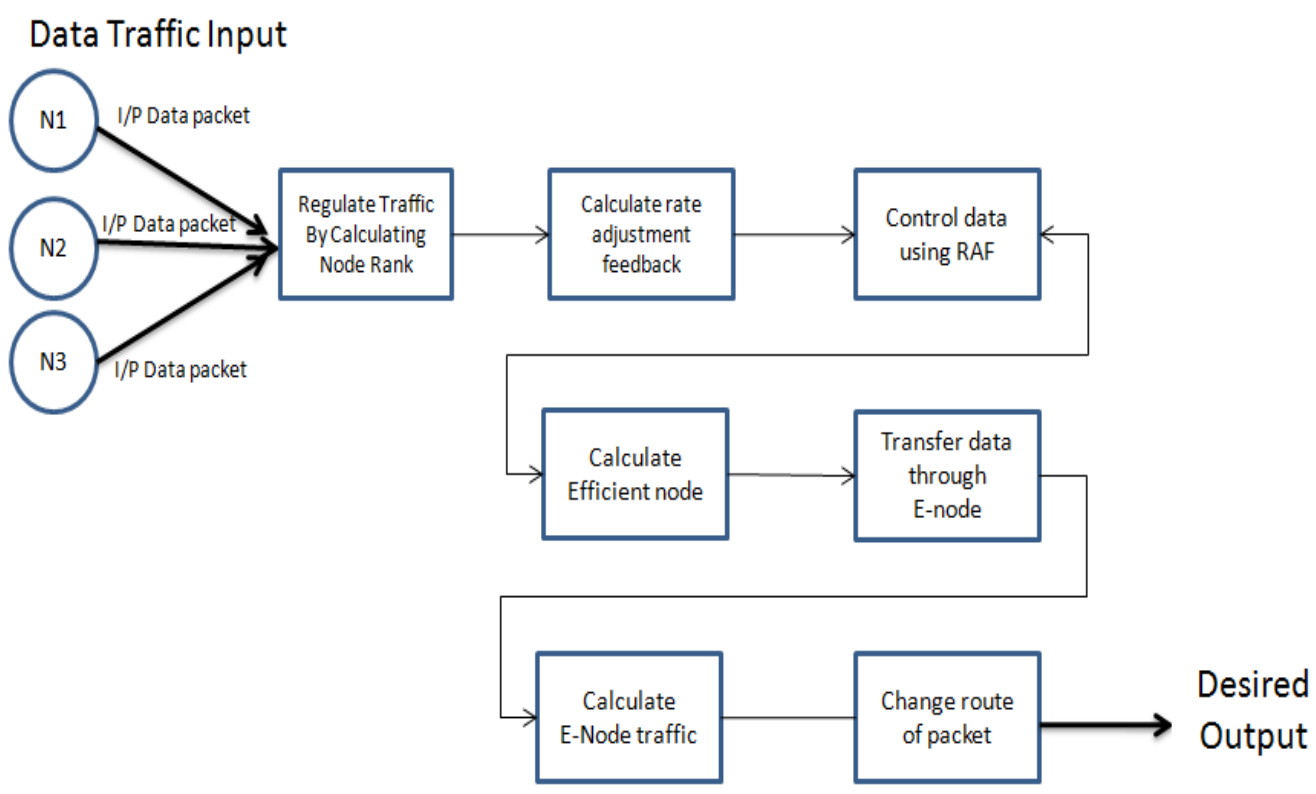

Figure 2. Traffic Contention and Control Mechanism

The wireless sensor network consists of multiple nodes between the source and destination [23]. This can consume a lot of energy and transmission delay if the data packet hops through every node in between. Hence, nodes with higher range and higher residual battery level are selected and marked as efficient nodes (e-node). The data is passed through these e-nodes to reduce hop counts and minimize the transmission delay. 


\subsection{Congestion control algorithms}

The existing congestion control approaches use either traffic control or resource control strategy to solve the problem of congestion [10]. The proposed traffic contention and control system is mainly based on three protocols viz., Hop by Hop Rate Adjustment protocol (HHRA), Energy Efficient Data Transfer Protocol (EEDT) and Alternative Routing for Congestion Control protocol (ARCC). These protocols are developed one for each stage to improve the quality of service in WSN by minimizing congestion and improving energy efficiency. In [24], a detailed analysis on the various QoS aware protocols for wireless sensor networks has been presented.

\subsection{Hop by hop rate adjustment protocol (HHRA)}

The Hop by hop rate adjustment protocol eliminates the contention by adjusting the transmission rate at each node if congestion is encountered. The degree of congestion is based on Node Rank. Based on the calculated Rate Adjustment Feedback, the source will slow down the transmission rate and retransmits the data packet according to the new transmission rate. The algorithm for HHRA is given below:

Step 1: Each node receives data packets from the nodes of heterogeneous network.

Step 2: At every node, the transmission rate of data packets is adjusted by sensing the degree of congestion. Step 3: For each node,

Calculate Node Rank (NR) using buffer overhead, hop count and MAC -overhead.

a) If $\mathrm{NR}>$ threshold value

b) Set Congestion Bit=1

Step 4: Calculate the Rate Adjustment Feedback (RAF) based on the rank and send it to the source node. $\mathrm{RAF}=($ Arate/HC $)-\Sigma \mathrm{OHMACi}-\Sigma \mathrm{CHBRi}$ Where, Arate is Arrival rate of packets at successive node, $\mathrm{HC}$ is the Hop Count, OHMACi is the MAC overhead and CHBRi is the Channel Busy Ratio.

Step 5: The value of RAF is propagated towards upstream nodes.

Step 6: The upstream nodes adjust their Transmission Rate as the New transmission rate (NRate), NRate $=$ TRate - RAF, Where TRate is the Present transmission rate

Step 7: The nodes retransmit the data packets according to the calculated NRate and avoids congestion.

\subsection{Energy efficient data transfer protocol (EEDT)}

The EEDT protocol improves energy efficiency by transmitting the data faster in minimum hop counts using efficient nodes (e-node). The efficient nodes have higher optimal coverage and residual battery level [25]. The data packets are transmitted only through these e-nodes selected within a given range [8]. The e-node sends an acknowledgment for each transmission and the transmitting node is put to a halt state until it receives feedback from the e-node. This approach enhances the quality of service, as the channel of the network is contention free and highly reliable. The algorithm for EEDT is given below,

Step 1: Identify the e-nodes in each range for transmitting packets using minimum number of hops.

Step 2: Find the e-node by sensing the energy level of available nodes in a given area.

Step 3: The node with highest Energy Level (EL) is selected as efficient node for that particular region.

Step 4: The EL of a node is the ratio of the Residual Energy at update interval to the Initial energy of the node.

Step 5: For a given region, the weight value assigned to a sensor node is based on the residual energy level of a sensor is given as, $\mathrm{W}(\mathrm{Ri})=\mathrm{EL} * \mathrm{~A}(\mathrm{Ri})$, Where $\mathrm{Ri}$ is the sensing region and $\mathrm{A}(\mathrm{Ri})$ is the area of sensing region $\mathrm{Ri}$.

Hence, for every iteration, the EEDT algorithm selects one e-node from the unselected sensor nodes, which covers the largest area with the highest residual energy level. Thus, the EEDT algorithm provides highly reliable data transmission in wireless sensor networks.

\subsection{Alternative routing for congestion control protocol (ARCC)}

When the data packets are transmitted through the same e-nodes there is a possibility of congestion in the e-nodes itself. Hence, in this situation, HHRA protocol is called to set the congestion bit and the route of the data packets are changed. Therefore, when congestion occurs in e-node, the alternative routing Congestion Control protocol (ARCC) is applied to provide reliable transmission without contention.

The algorithm for ARCC is given below,

Step 1: For each Cluster Head node, Set candidate node $=0$

Step 2: If current node $=$ Cluster Head node Set hop count $=$ hop count +1 Else Set hop count $=0$

Step 3: Set Candidate node $=$ Candidate node +1

Step 4: If Current_node receives ack with congestion bit set, Update candidate_parent table

Step 5: Search candidate_parent table and find node_id with min (congestion bit) and send data packet.

Step 6: If current_node receives congestion_update_msg, Update candidate_parent table Else if current_node receives data packet and accept it Set buffer $=$ buffer +1

Step 7: If buffer = full, Update congestion bit Send ack_pkt with congestion bit information 
Hence the proposed ARCC algorithm alters the route of the data packet to avoid congestion and ensure successful packet transmission with high reliability.

\section{SIMULATIONS RESULTS}

The proposed HHRA, EEDT and ARCC are simulated in Network Simulator 2 (NS2). For experimentation purposes, the wireless sensor network comprises 100 nodes and the data flow in the network is simulated. The proposed HHRA algorithm is compared with the existing Dynamic Contention Window based Congestion Control (DCWCC) algorithm and the performance is analyzed. The following are the metrics that are considered for analyzing the performance of the proposed algorithms.

\subsection{Performance metrics}

To prove the validation of HHRA, EEDT and ARCC algorithms we have considered the following metrics. The deployed sensor nodes and their range is shown in Figure 3.

Aggregated Throughput: It is the ratio of the number of packets transmitted to the number of packets received. Average Energy Consumption: It is defined as the utilization of energy consumed by the nodes for Sending and receiving a data packet.

Packet Delivery Ratio: It is defined as the ratio of the total number of packets transmitted from the Source to the total number of packets delivered at the sink.

Average Packet Loss: It is defined as the average number of packets lost during transmission.

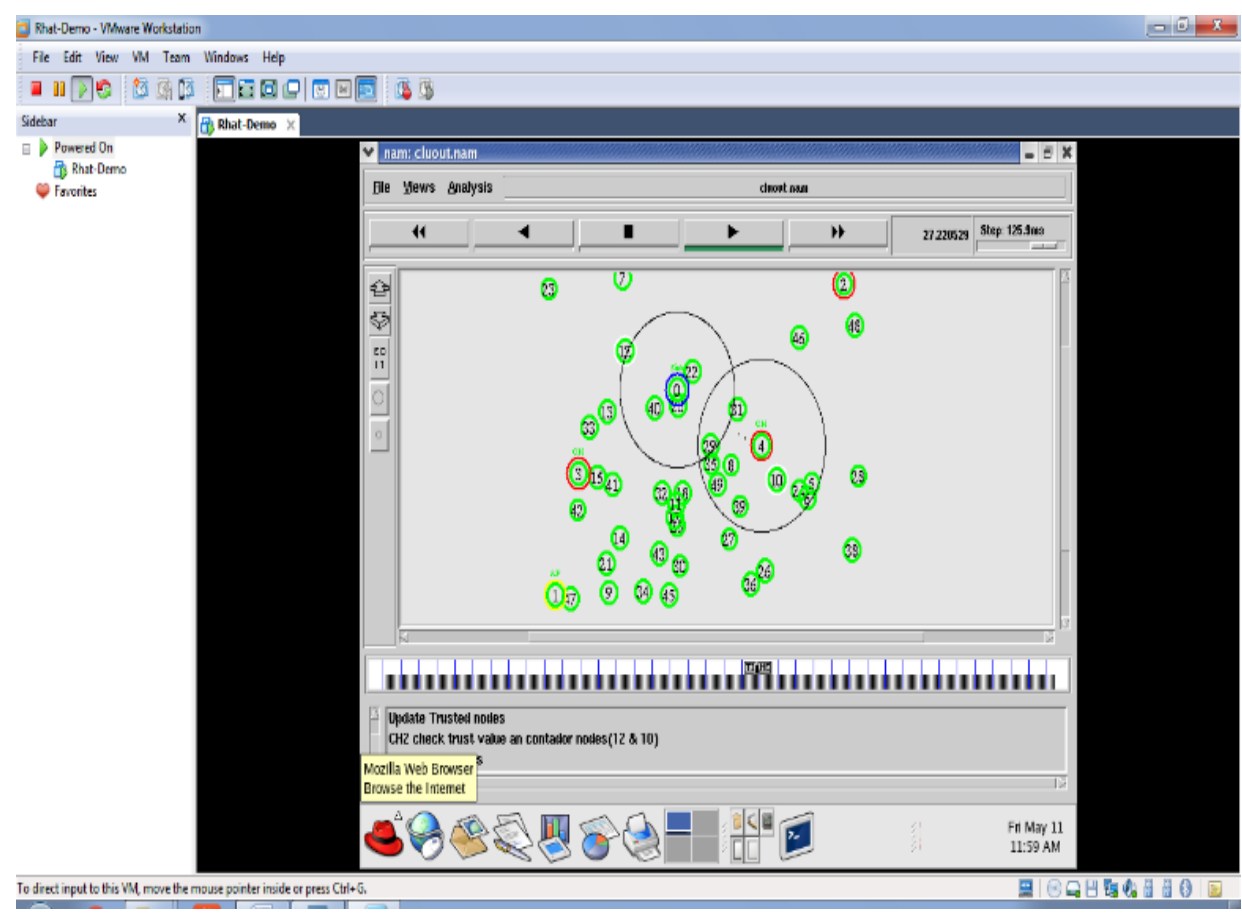

Figure 3. Set up of nodes in wireless sensor network

The HHRA protocol execution is validated by the differing number of nodes and by shifting the transmission rate. Keeping in mind the end goal to test the versatility, the execution of the conventions by shifting the quantity of nodes as $25,50,75$ and 100 is estimated. The normal energy consumption of the nodes increments as the quantity of nodes increments from 15 to 100. But, in HHRA protocol, the general energy consumption is reduced in all cases compared to DCWCC as shown in Figure 4.

The Packet Delivery Ratio (PDR) is computed for both HHRA and DCWCC algorithms. It is evident from Figure 5, that when the number of nodes is increased, the hop counts between source and destination is also increased which leads to an increase in packet delivery ratio. The packet delivery ratio of DCWCC gradually decreases as there is an increase in the number of nodes. But, the PDR of HHRA is comparatively higher than DCWCC. 


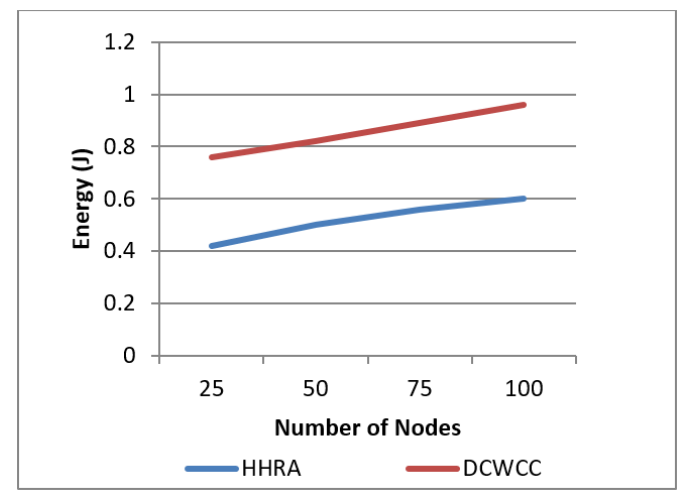

Figure 4. Average energy consumption of nodes

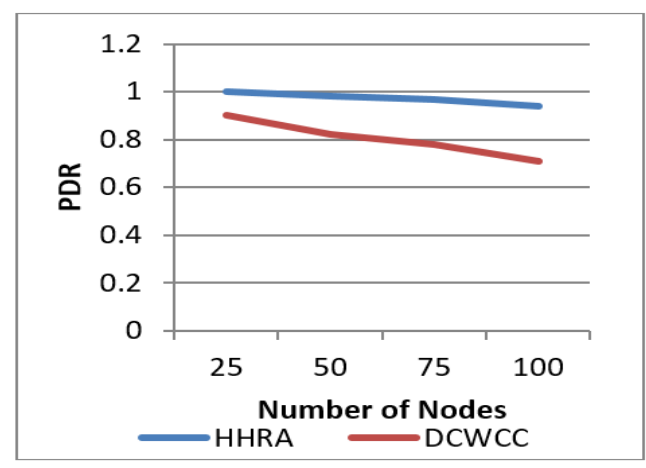

Figure 5. Packet delivery ratio

The throughput of the existing DCWCC is compared with HHRA algorithm and is depicted in Figure 6. The throughput of HHRA is higher than DCWCC since the congestion is immediately handled by calculating the RAF. The performance of the proposed EEDT protocol is compared to the existing MAC Aware Energy Efficient Reliable Protocol (MAEERP).

To study the impact of the increase in the number of source nodes, the number of sources is varied from 2, 4, 6 and 8 and the performance of the EEDT and MAEERP protocols is analyzed. It is understood from Figure 7, that the number of packets lost when the source is altered is high in MAEETP than EEDT. The average energy consumed by the nodes during transmission is shown in Figure 8 . It is obvious that energy consumption shows a steep increase with respect to the number of source nodes.

As the number of source nodes is increased, the energy consumed in the existing MAEERP is very high compared to EEDT algorithm. This is due to the fact that, EEDT makes use of energy efficient scheduling and hence the energy consumption is decreased and almost stable even if the source nodes increase.

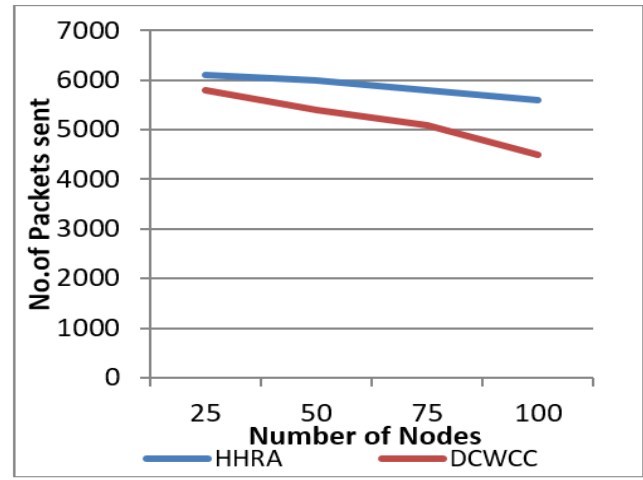

Figure 6. Aggregated throughput

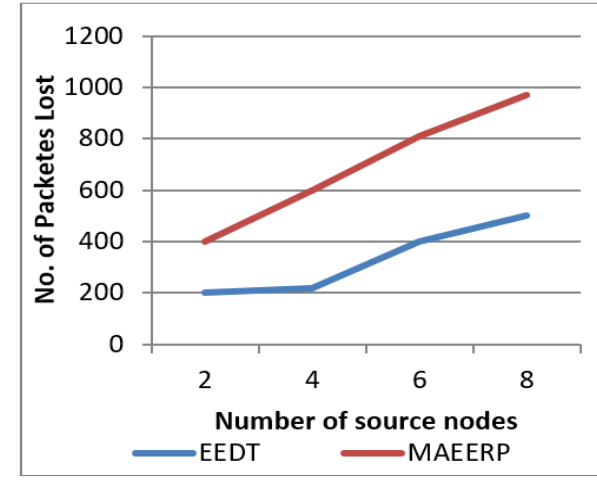

Figure 7. Packet loss

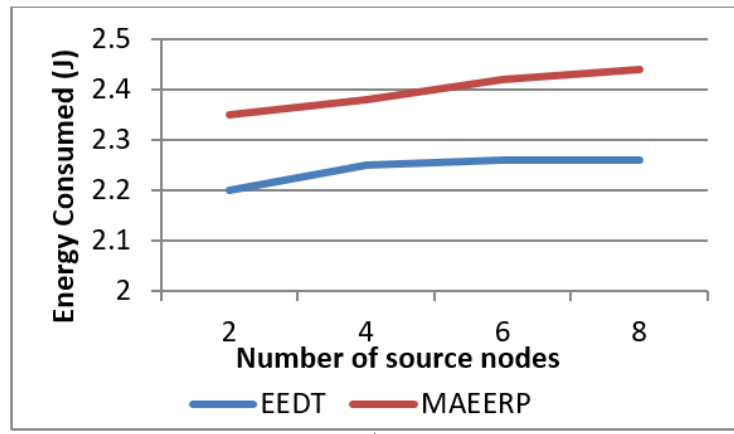

Figure 8. Energy consumption for multiple source nodes 


\section{CONCLUSIONS}

In wireless sensor networks, the cooperation between nodes is stimulated by only considering a single objective (e.g., energy consumption or network lifetime). This approach is not adaptive to the variation of demand for topology performance. To overcome this issue, an Effective Traffic Contention and Control Mechanism is proposed for improving the Quality of Service in Wireless Sensor Networks. In this work, three different algorithms are implemented to solve the challenges such as congestion, quality of service, battery optimization and link lifetime. The congestion degree is sensed at each node and transmission rate is adjusted accordingly to avoid congestion. The data packets are sent through specific efficient nodes to minimize the transmission delay. And if there is any congestion in the path of the packet, the alternative route is selected to transfer the data packet to the sink node. Therefore, all these algorithms are implemented to ensure congestion-free data transfer with the high quality of service and minimum transmission delay. In the future, algorithms can be developed to increase the utilization of bandwidth, removal of redundant data and also to handle the buffer size limitation.

\section{REFERENCES}

[1] Mian Ahmad Jan, Syed Rooh Jan, Muhammad Alam, Adnan Akhumzada \& Izaz Ur Rahman, “A Comprehensive Analysis of Congestion Control Protocols in Wireless Sensor Networks", Journal of Mobile Networks and Applications, Vol. 23, No. 3, pp. 456-468, 2018.

[2] Ketshabetswe, L.K., Zungeru, A.M., Mangwala, M., Chuma \& J.M., Sigweni, B, "Communication protocols for wireless sensor networks: a survey and comparison”, Heliyon, Vol. 5, No. 5, pp. 1-43, 2019.

[3] Muwonge Ssajjabbi Bernard, Tingrui Pei \& Kimbugwe Nasser "QoS Strategies for Wireless Multimedia Sensor Networks in the Context of IoT at the MAC Layer, Application Layer, and Cross-Layer Algorithms", Journal of Computer Networks and Communications, pp. 1-34, 2019.

[4] Tarek Azizi \& Rachid Beghdad, "Increasing QoS Parameters in WSNs through Spiral-Based Clustered Architecture", The 7th International Conference on Ambient Systems, Networks and Technologies, Procedia Computer Science, Vol. 83, pp. 401-408, 2016.

[5] Seddiki Noureddine, Benahmed Khelifa \& Belgachi Mohammed, "Approach to minimizing consumption of energy in wireless sensor networks", International Journal of Electrical and Computer Engineering, Vol. 10, No. 3, pp. 2551-2561, 2020.

[6] Mahdi, O.Adil and Al-Mayouf, Y.R Bahar and Ghazi, Abed and Wahab, A.W Abdul and Idris, M.Y Idna, "An Energy- Aware and Load-balancing Routing scheme for Wireless Sensor Networks", Indonesian Journal of Electrical Engineering and Computer Science (IJEECS), Vol. 12, No. 3, pp. 1312-1319, 2018.

[7] Madhu Patil \& Chirag Sharma, "Energy Efficient WSN by Optimizing the Packet Failure in Network", Indonesian Journal of Electrical Engineering and Computer Science (IJEECS), Vol. 7, No. 2, pp. 415-425, 2017.

[8] Mohamed Abdelaal, Oliver Theel, Christian Kuka \& Peilin Zhang, "Improving Energy Efficiency in QoSConstrained Wireless Sensor Networks”, International Journal of Distributed Sensor Networks, pp. 1-28, 2016.

[9] Parvinder Singh \& Rajeshwar Singh, "Energy-Efficient QoS-Aware Intelligent Hybrid Clustered Routing Protocol for Wireless Sensor Networks", Journal of Sensors, pp. 1-13, 2019.

[10] James DzisiGadze, DelaliKwasiDake \& Kwasi Diawuo, "Adaptive Congestion Control Protocol for Wireless Sensor Networks", International Journal of Wireless \& Mobile Networks, Vol. 5, No. 5, pp. 1-16, 2013.

[11] T. Phatak and S. D. Sawarkar, "Enhancing QoS of Wireless Sensor Network by detection of faulty sensor node", International Conference on Computing, Analytics and Security Trends, pp. 100-105, 2016.

[12] S. M. Jameii, K. Faez \& M. Dehghan, "AMOF: Adaptive Multi-objective Optimization Framework for Coverage and Topology Control in Heterogeneous Wireless Sensor networks", Telecommunication Systems, Vol. 61, No. 3, pp. 1-16, 2015.

[13] J. Gui \& Z. Zeng, "Joint Network Lifetime and Delay Optimization for Topology Control in Heterogeneous Wireless Multi-Hop Networks", Journal of Computer Communication, Vol. 59, Issue C, pp. 24-36, 2015.

[14] Quiming Liu, Xueijing Jiang \& Yang Zhou, "Per-Flow End-To-End Delay Bounds in Heterogeneous Wireless Networks", 3rd IEEE international Conference on Computer and Communication, China, pp. 295-299, 2017.

[15] Z.Chen, A.Li, Z.Li, Y-J. Choi, H.Sekiya, \& J.Li, "Energy Efficient Broadcasting Scheme for Smart Industrial Wireless Sensor Networks", Mobile Information Systems, Vol. 20, pp. 1-17, 2017.

[16] R. Sumathi \& R. Srinivasan, "QoS aware routing protocol to improve reliability for prioritised heterogeneous traffic in wireless sensor network", International Journal of Parallel, Emergent and Distributed Systems, Vol. 27, No. 2, pp. 143-168, 2012.

[17] Zeynab Bidoni \& Roy George, "Network Service Quality Rank: A Network Selection Algorithm for Heterogeneous Wireless Networks", IEEE Symposium on Architectures for Networking and Communication Systems, USA, pp. 239-240, 2014.

[18] J. S. Gui \& K. Zhou, "Flexible Adjustments Between Energy and Capacity for Topology Control in Heterogeneous Wireless Multi-Hop Networks", Journal of Networks and Systems Management, Vol. 24, No. 4, pp. 789-812, 2016.

[19] Ali Ghaffari, "Congestion control mechanisms in wireless sensor networks: A survey", Journal of Network and Computer Applications, Vol. 52, pp. 101-115, 2015.

[20] Vikas Srivastava, Karan Singh \& Sachin Tripathi, "A Critical Insight into Congestion Control Mechanism in WSN”, International Journal of Multimedia and Ubiquitous Engineering, Vol. 12, No. 1, pp. 269-280, 2017. 
[21] Nadia A. Shiltagh \& Zainab G. Faisal, "Traffic Management in Wireless Sensor Network Based on Modified Neural Networks", Iraqi Journal for Computers and Informatics, Vol. 1, No. 1, pp. 2-6, 2014.

[22] Daniela Aguirre-Guerrero, Ricardo Marcelín, Enrique Rodriguez \& Michael Pascoe, "Congestion Control for a Fair Packet Delivery in WSN: From a Complex System Perspective”, The Scientific World Journal, pp. 1-13, 2014.

[23] M. Bhardwaj, T. Garnett \& A. P. Chandrakasan, "Upper bounds on the lifetime of sensor networks", IEEE International Conference on Communication (ICC), Finland, pp. 1-37, 2001.

[24] M. Aykut Yigitel, Ozlem Durmaz Incel \& Cem Ersoy, "QoS-aware MAC protocols for wireless sensor networks: A survey”, Computer Networks, Vol. 55, No. 8, pp. 1982-2004, 2011.

[25] Shuang Zhai, Yu FU, Chao Cheng \& Zhihong Qian, "An Energy-Efficient Clustering Algorithm for Heterogeneous Wireless Sensor Networks", IEEE International Conference on Communications in China (ICCC), pp. 1-9, 2017.

\section{BIOGRAPHIES OF AUTHORS}

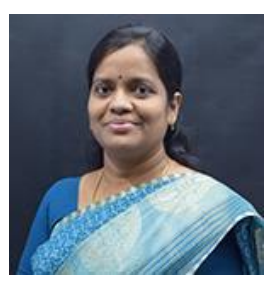

Dr.G. Kavitha is presently working as an Associate Professor in the Department of Information Technology, B.S.A. Crescent Institute of Science \& Technology. She obtained her Master's Degree in Computer Science and Engineering from Madras Univerisity in 2002 and Doctor of Philosophy in Faculty of Information and communication, Anna University in 2013. Her research interests focus on workflows on the Grid and Cloud Computing, Wireless Sensor Networks and Artificial Intelligence.

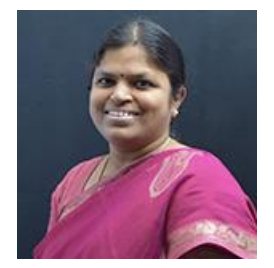

Dr. P. Latchoumy obtained her master's degree from Anna University in 2005 and Doctor of Philosophy from B.S.A. Crescent Institute of Science \& Technology in 2018. Presently, she is working as an Associate Professor in the Department of Information Technology, B. S. Abdur Rahman Crescent Institute of Science and Technology. Her research interest focuses on failure handling in grid and cloud computing, Wireless Networks, and data science.

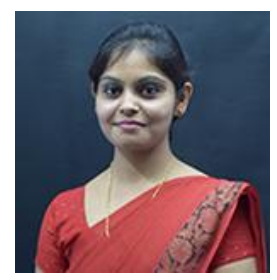

Ms.A. Sonya is presently working as an Assistant Professor in the Department of Information Technology, B.S.A. Crescent Institute of Science \& Technology. She obtained her Master's Degree in Computer and Communication in 2012. She is pursuing her doctoral degree in the Department of IT, B.S.A. Crescent Institute of Science \& Technology. Her research interests include Cloud Computing, Soft Computing and Wireless Networks. 